\title{
The lack of uniformity of the deformations and the degree of deforming in case of orbital deformation
}

\author{
Sorin-Dumitru Grozav ${ }^{1 *}$, Gabriel Nicodim Ciuşcă ${ }^{1}$ and Vasile Ceclan ${ }^{1}$ \\ ${ }^{1}$ Technical University of Cluj Napoca, Manufacturing Engineering Department, ROMANIA
}

\begin{abstract}
The degree of deformation and the lack of uniformity of the deformations are two important factors orbital deformity. The degrees of deformation in the case of orbital oscillating deformation between 40 and $70 \%$, is much higher than in conventional methods. Two other important parameters that may change unevenness strains are axial force and advance. Their influence can be seen from the diagrams unevenness, and hence the higher the value of these parameters is reduced unevenness strains.
\end{abstract}

\section{Introduction}

Orbital deforming it is an unconventional forming technology of metals, having as a particular the fact that applying in a convenient way the forming pressure; it is obtained a considerable reduction of forming pressure for the same forming straight. The principle of orbital deforming was patented in 1910, but his extension began in the last 25 - 30 year, after the apparition of the machines able to realization of this operations. This method is based on the principle that the pressure is not applied on the whole surface of the work piece, and it is applied only a $20-30$ of it. This it is show in the figure 1 [1], [2], [3].

The pressure application zone is variable in time, because the pressure is moving on the surface of the work piece with a constant frequency of $150-200$ cycles $/ \mathrm{min}$. The most important traits of this method, having as result a sequential penetration of the pressure in every parts of the work piece surface are: the wobbling motion of the tool together with the superior tool reported to the vertical axes, and the four moves of that.

The determination of the tools track which is the most suitable for the work piece is important for metal flow in the tool. A similar influence has the choice of the wobbling limit angle has the same influence as forming pressure.

The pressure application zone is variable in time, because the pressure is moving on the surface of the work piece with a constant frequency of $150-200 \mathrm{cycles} / \mathrm{min}$. The most important traits of this method, having as result a sequential penetration of the pressure in every parts of the work piece surface are: the wobbling motion of the tool together with the superior tool reported to the vertical axes, and the four moves of that [2], [3], [4], [5].

${ }^{*}$ Corresponding author: sorin.grozav@,tcm.utluj.ro 


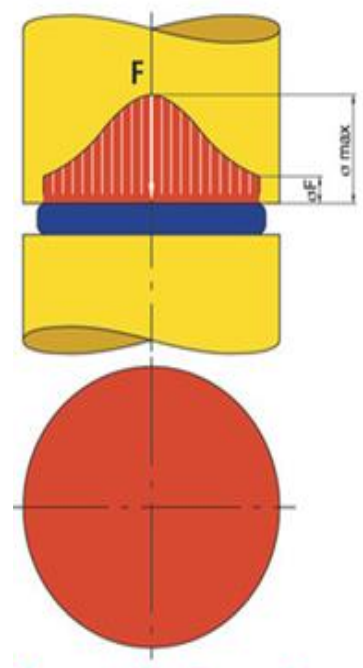

Normal deformation

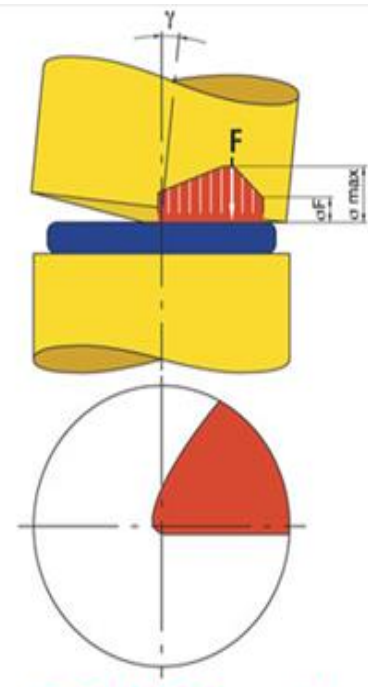

Orbital deformation

Fig. 1. Principle orbital deformation.

The determination of the tools track which is the most suitable for the work piece is important for metal flow in the tool. A similar influence has the choice of the wobbling limit angle has the same influence as forming pressure.

If the deformation of the conventional, inter-acting with the tool on its entire front surface of preform, when deformation orbital focus of deformation is located at any stage of the process (the shaded area in figure 1). The initial preform, having usually formed of a cylinder or ring, is seated in lower die which is vertically oriented axis of symmetry. The upper die having the conical shape of the roller is inclined from the vertical by an angle $\theta$ (figure 2) [6].

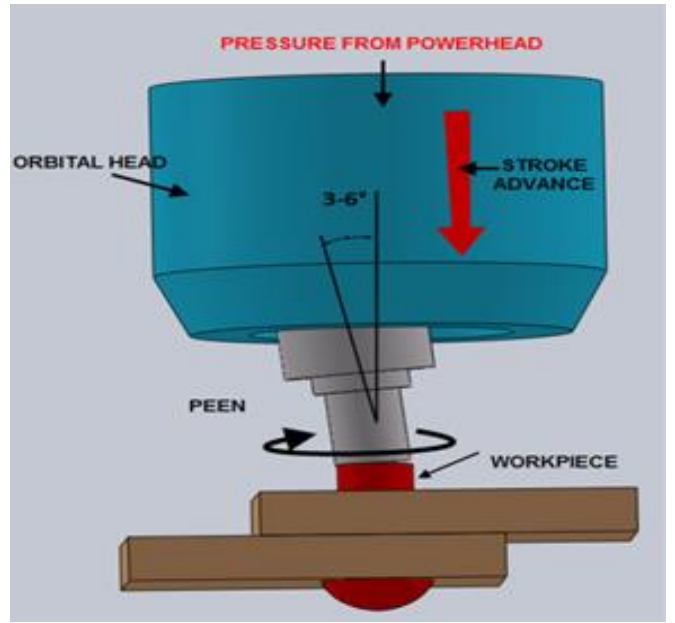

Fig. 2. Realization rivets by orbital deformation [6].

The upper die must be so positioned that the apex of the cone to be located always on the axis of symmetry of the lower die, which is the central axis of rotation. Changing the shape of the preform is carried out by oscillating the upper die around the central axis of rotation and the vertical movement of advance, either the upper die to the lower die, or vice 
versa. In any case, the tip of the cone, which is always fixed, becomes the pivot point of the upper die. Kinematic point of view it is a rigid fixed. Thus, at any given time a portion of the volume part is in the focus of deformation. Due to the oscillating movement of the upper mould and the advance due to axial deformation zone is dynamic. It moves the propeller downward sweep the front surface of the work-piece and approaching nearer to base the preform. Penetration depth of deformation on the preform height depends specifically track configuration and the actual amount of process specific parameters.

\section{The lack of uniformity of the deformations and the degree of deforming}

\subsection{Experimental research}

In order to be able to operate with maximum efficiency the facilities offered by this new and wider technology, it is necessary to study the flow of the material and the distribution of deformations in the volume of the orbital deformed blank, necessary for the design of a correct orbital deformation technology. [1], [2], [7], [8] For the study of the unevenness of the deformations at the orbital deformation we worked with lead cylindrical specimens with different ratios between height $(\mathrm{H})$ and diameter $(\mathrm{D})$ : H / D $=0.2 \ldots 2$. We have drawn on the lateral surface a network of equidistant lines parallel to the base of the specimens, and on the front surface they have drawn equidistant concentric circles. For the modification of the parameters of the networks after the orbital deformation we could characterize the distribution of the deformations and the flow of the material.

The experiments were performed on a Polish orbital PXW100 deforming machine, which can develop a $1600 \mathrm{KN}$ axial force $\left(\mathrm{F}_{\mathrm{a}}\right)$ with an oscillating angle of the upper tool of $\gamma=2^{0}$ at an oscillation frequency of 200 oscillations / min. This press carries out four types of movements. [1], [2], [4]

The recording of the run-time parameters was performed on a $\mathrm{x}-\mathrm{y}$ inscriber, and the force was determined from the pressure of the hydraulic cylinder read on the manometer. The specimens were orbital deformed, with varying degrees of deformation, using multiple values $x-y$ of deformation force and axial feed (a). The obtained results were compared with those from the conventional deformation of identical specimens. Following the modification of the geometric shape of the deformed specimens, it is observed that, unlike the classic deformation in which the specimen is the shape of the barrel, the orbital deformation takes the shape of the fungus, having the maximum diameter in the upper part and not in the middle of the height.

\subsection{Experimental results}

The degree of deforming is calculated with the equation:

$$
\varphi_{h 1}=\frac{\Delta h}{h}
$$

where: $\Delta \mathrm{h}$ is the height variation after the repression and $\mathrm{h}$ is the initial height of the semifinished product. 
In the figure 3.a) you can see that for higher values of the rapport $h / d$, the more powerful deforming is situated at the superior part of the semi-finished product, while at the smaller values of the rapport, the inferior part of the semi-finished product suffers a more powerful deforming. This phenomenon is caused by the passage of the material from the side part to the lower one, fact that leads to the diminishing of the side network distances. The middle area of the semi-finished product is a little deformed and we can say that it doesn't depend on the rapport $\mathrm{h} / \mathrm{d}$.

The degree of radial deforming can be expressed by the equation:

$$
\varphi_{r} \frac{\Delta R}{R}
$$

where: $\Delta \mathrm{R}$ is the changing of the cylinder radius and $\mathrm{R}$ is its initial radius.

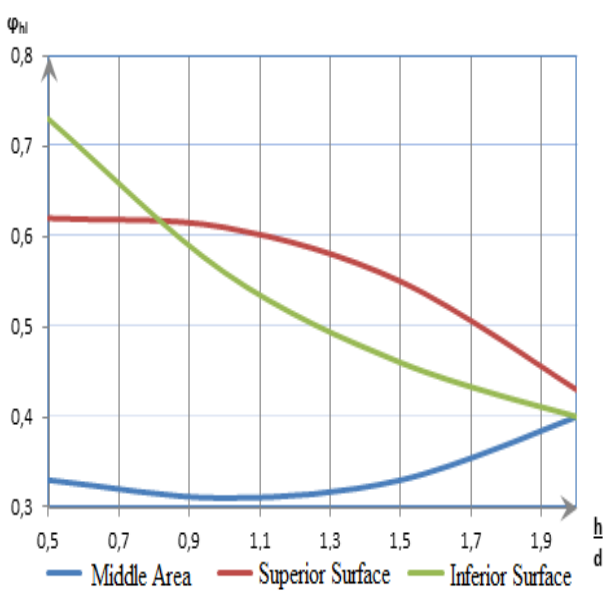

a)

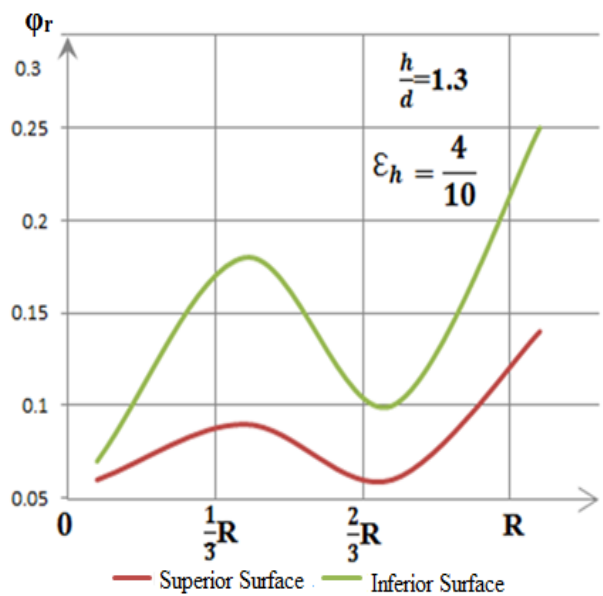

b)

Fig. 3. The variation of the deforming grade: a) in height; b) radial.

In the figure 3.b) there is represented the variation of the radial deforming degree. From the figure you can see that on the inferior surface of the semi-finished product the distribution of the radial deforming is similar to the one from the conventional repression, having a stationary middle area, respective an edge area with a maximum deforming. But, on the inferior surface, the repartition of the deforming has as a distinctive particularity the appearance of a medium deforming, even in the central area.

The knowing of the degree of unevenness of the deformations has a special importance and it can be express by the rapport:

$$
\varphi_{d u}=\frac{d_{m s x}}{d_{\min }}
$$

Where $d_{\max }$ and $d_{\min }$ are the maximum and the minimum diameters of the deformed semi-finished product (figure 4). 
The dependence of the unevenness of the deformations according to the degree of deformation in height, for different rapports $h / d$ is presented in the figure $4 . a$ ) and in the figure 4.b) there is presented the dependency of the unevenness degree towards the rapport $\mathrm{h} / \mathrm{d}$ at the same deforming grade. From this diagram results that the unevenness of the deformations increases almost linear till the maximum value reached by the deforming degree between $0.4 \ldots 0.5$, afterwards decreasing slowly. The unevenness is more accentuated for higher values of the rapport $\mathrm{h} / \mathrm{d}$.

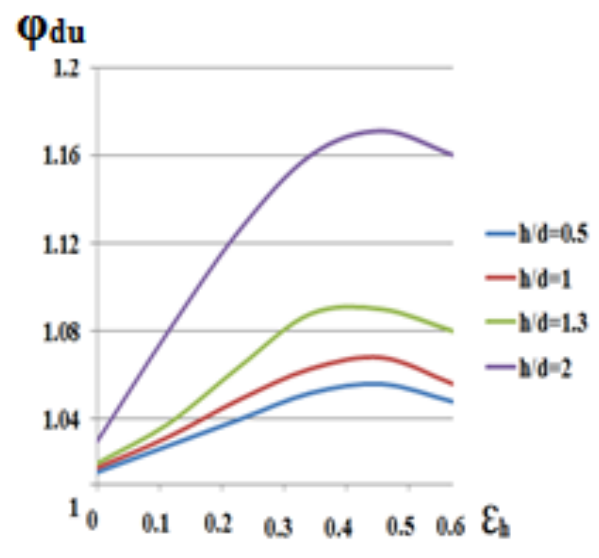

a.

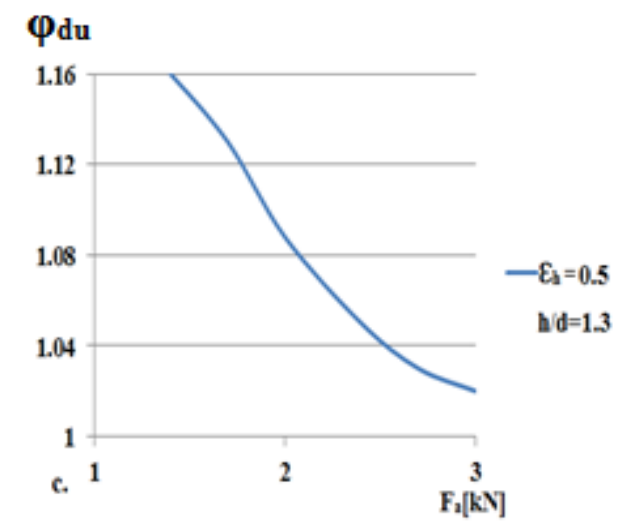

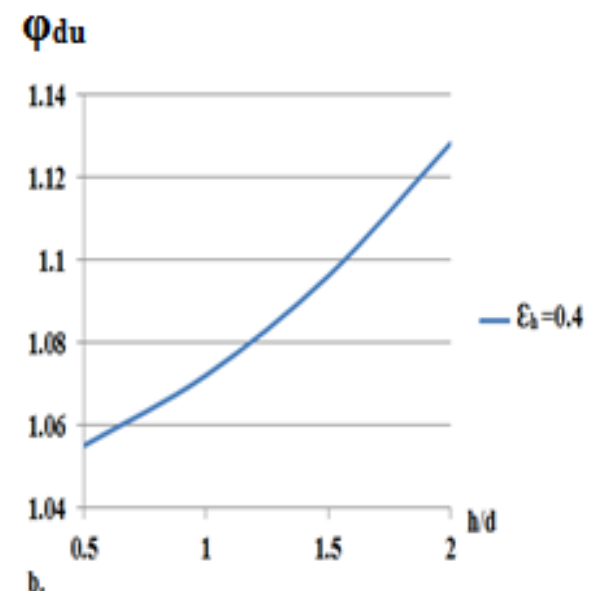

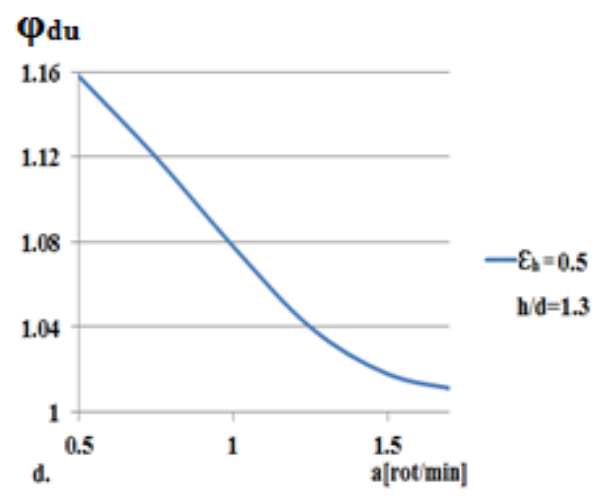

Fig. 4. The evolution of the unevenness degree of the deformations $\varphi_{\mathrm{du}}$ : a - according to the deforming degree on height; $\mathrm{b}$ - according to the rapport $\mathrm{h} / \mathrm{d} ; \mathrm{c}-$ according to the axial force; $\mathrm{d}-$ according to the advance.

Other 2 important parameters which can modify the unevenness of the deformations are the axial force and the advance. Their influence upon the unevenness can be observed in the figure 4,c and $d$, from where results that, together with the increasing of these parameters, the unevenness of the deformations is reduced. 


\section{Conclusion}

Following the analysis of the results presented in the diagrams presented, some conclusions are drawn which are very important for the establishment of a correct orbital deformation technology:

- at the orbital deformation of the pieces with different outer diameters, the maximum diameter will be located at the upper part because the unevenness of the deformations will help to fill the moulds;

- at large diameter differences the force and the axial feed will be reduced in order to increase the unevenness of the deformations;

- the parts with a constant outer diameter, which are desirable for the smallest unevenness of the deformations, will be deformed with a maximum axial force and advance provided by the deforming machine;

- degree of deformation in orbital deformation oscillating between 40 and $70 \%$, is much higher than in conventional methods;

- geometrical format of the contact surfaces in orbital deformation directly the lack of uniformity of the deformations and the degree of deforming.

\section{References}

1. S. D. Grozav, Studies and researches in process parameters variation in Rotary Forging, PhD Thesis, University of Cluj-Napoca (1995)

2. S. D. Grozav, Orbital Deformation MEDIAMIRA Publishing House, Cluj-Napoca, Engineering Collection, ISBN 978-973-713-237 (2009)

3. P.M. Standrig, Investigation into Rotary Forging, Ph.D. Thesis University of Nottingham (1982)

4. S. D. Grozav, A. Crai, Annals of the Oradea University, ISSN 1583-0691 (2006)

5. S. D. Grozav, Gh. Gligor, Annals of MTeM, 175 -178, ISBN 973-9087-83-3 (2007)

6. $* * *$ http://msvs-dei.vlabs.ac.in/Orbital.php

7. T. Canta, I. Dragan, Gh. Achimas, Constr. de Maşini, 39, 2-3, 101-104 (1987)

8 Drăgan, T. Canta, I. Nistor, Gh. Goron, D. Săbăduş, Constr. de Maşini, 41, 2-3, 100-103 (1989) 